\title{
LES of a Methanol Spray Flame with a Stochastic Sub-grid Model
}

\author{
W. P. Jones, A. J. Marquis, Dongwon Noh* \\ Department of Mechanical Engineering, Imperial College London, Exhibition Road, London SW7 2AZ, UK
}

\begin{abstract}
This paper describes the Large Eddy Simulation (LES) of a methanol/air turbulent nonpremixed spray flame. An Eulerian stochastic field method is employed for the turbulence-chemistry interaction of the gas phase while a Lagrangian formulation is used for the liquid phase. A reduced reaction mechanism (18 species and 14 reactions) is adopted and stochastic models are used to account for the influence of sub-grid scale ( $\mathrm{sgs}$ ) motions on droplet dispersion and evaporation. Comparisons of the predicted gas phase and droplet statistics with measurements show a good agreement confirming that the droplet dispersion and evaporation models used in this work are adequate. The general features of the spray flame such as the occurrence of external group combustion and its development into separate combusting islands are well captured.
\end{abstract}

Keywords:

Large Eddy Simulation, Turbulent Spray Combustion, Stochastic field method, PDF method

\section{Introduction}

Combustion systems with liquid fuel sprays have been extensively used in various engineering applications such as industrial furnaces, internal combustion engines, rocket propulsion and gas turbines. The modelling of such systems requires a good understanding of both physical and chemical processes taking place in these two phase flows. However, the numerical simulation of liquid spray flames is challenging because it is difficult to simulate their multi-scale nature. The ability to model and simulate particle-laden flows could result in significant advances in the design of various spray combustion systems in terms of their efficiency and reduction in pollutant emissions.

LES is an effective tool for predicting the properties of turbulent reactive flows. In LES, the equations of motion are solved directly for the large-scale energetic turbulent motions while the influence of the sub-grid scale $(s g s)$ motions on the resolved flow field are modelled. In the present work, the liquid phase is described using a Lagrangian formulation whilst an Eulerian approach is employed for the gas phase. In the context of LES, the size of droplets is assumed to be sufficiently small

\footnotetext{
${ }^{*}$ Corresponding author Noh )

Email address: dongwon.noh10@imperial.ac.uk (Dongwon

Preprint submitted to Proceedings of the Combustion Institute
}

so that they can be treated as point sources of mass, momentum and energy. Stochastic models are used to account for the influence of sgs motions on particle dispersion, i.e. acceleration and particle vaporisation. Previous studies have investigated different experimental configurations, e.g. a swirl stabilised burner with liquid kerosene [1], an aeronautical type combustor, which is the DLR Generic Single Sector Combustor, [2] and a methanol fuelled spray burner in a hot coflow experiencing turbulent auto-ignition [3].

The overall aim of the present research is to conduct LES simulations of a turbulent spray flame possessing multiple length and time scales involving chemical reactions and interactions between gas phase turbulence and particle motions. The predictive capabilities of LES with major improvements regarding liquid phase modelling are validated by reproducing the turbulent flame structures of an experimental burner [4]. The spray flame configuration studied in the present paper has also been the subject of a previous numerical study [5]. A Reynolds averaged approach was adopted with a Reynolds stress transport equation model being used in conjunction with a probability density function $(p d f)$ equation method. Account was taken of droplet evaporation and two-way coupling of mass and momentum exchange. It appears, however, that the present work represents a first attempt at applying LES to the config- 
uration under consideration.

The structure of this paper is as follows. First, the experimental apparatus investigated in the current work is described. The mathematical formulation of the LES filtered equations for physical variables of the continuous phase flow and the methods used to predict the particle dispersion and evaporation in a stochastic manner are presented. The numerical set-up and initial/boundary conditions are then explained. The numerical results are compared with experimental measurements and discussed in detail. The conclusions of the current investigation are made in the last section.

\section{Experimental Configuration}

The experimental burner is a canonical turbulent methanol/air spray flame designed by Karpetis and Gomez [4]. The liquid fuel, methanol, is fed into the burner housing using a commercial ultrasonic atomizer (Sono-Tek) which produces particles at minimal velocity. The drops are carried upwards by air through a number of primary holes. Furthermore, around $4 \%$ of the total air flow rate is diverted around the atomizer in order to carry the drops past its tip and create a small region of recirculation where the flame is attached.

In the experiments, two methanol spray flames with Reynolds numbers based on cold conditions between 21,000 and 28,000 are examined and referred to as flame I and II respectively; in the present work, only flame I is examined. The total mass flow rates of air and methanol are $1.6 \times 10^{-3} \mathrm{~kg} / \mathrm{s}$ and $8.5 \times 10^{-5} \mathrm{~kg} / \mathrm{s}$ respectively. The measurements are performed at $0.1 \mathrm{D}, 0.5 \mathrm{D}$ and at every half burner exit diameter $(\mathrm{D}=12.7 \mathrm{~mm})$ thereafter up to $6.5 \mathrm{D}$ in the axial direction. Phase Doppler Anemometry (PDA) is used to measure spray properties, i.e. droplet number density and size and velocity statistics. Chemiluminescence imaging and Raman spectroscopy are adopted to obtain the spray flame images and the gas phase temperature respectively.

\section{Numerical Modelling}

\subsection{Filtered Gas Phase Equations}

The density-weighted filtered governing equations of low-Mach number, variable density flows with point sources of mass, momentum, species or energy contributions from the dispersed phase are:

$$
\begin{gathered}
\frac{\partial \bar{\rho}}{\partial t}+\frac{\partial \bar{\rho} \tilde{u}_{i}}{\partial x_{i}}=\overline{\dot{S}}_{\text {mass }} \\
\frac{\partial \bar{\rho} \tilde{u}_{i}}{\partial t}+\frac{\partial \bar{\rho} \tilde{u}_{i} \tilde{u}_{j}}{\partial x_{j}}=-\frac{\partial \bar{p}}{\partial x_{i}}+\frac{\partial \bar{\sigma}_{i j}}{\partial x_{j}}+\frac{\partial \tilde{\tau}_{i j}^{s g s}}{\partial x_{j}}+\overline{\dot{S}}_{m o m, i} \\
\frac{\partial \bar{\rho} \tilde{\phi}_{\alpha}}{\partial t}+\frac{\partial \bar{\rho} \tilde{\phi}_{\alpha} \tilde{u}_{j}}{\partial x_{j}}=\frac{\partial}{\partial x_{j}}\left(\frac{\mu}{\sigma} \frac{\partial \tilde{\phi}_{\alpha}}{\partial x_{j}}\right)-\frac{\partial \tilde{J}_{j}^{s g s}}{\partial x_{j}} \\
+\frac{\rho \dot{\omega}_{\alpha}(\underline{\phi}, T)}{\dot{S}_{\alpha}}
\end{gathered}
$$

where $\rho, u_{i}, p$ and $\phi_{\alpha}$ represent the fluid density, velocity vector, pressure and scalar quantities, i.e. species mass fraction and enthalpy. Equal diffusivities are assumed for the species and enthalpy equations so that $\sigma$ is the Schmidt and Prandtl number as appropriate.

A dynamic version of Smagorinsky model [6] is adopted to close the unresolved residual stresses $\tilde{\tau}_{i j}^{s g s}$. Equation (3) involves the sub-grid scalar flux $\tilde{J}_{j}^{s g s}$ and the filtered chemical source term $\overline{\rho \dot{\omega}_{\alpha}(\phi, T)}$ which represents the net formation rate of species $\alpha$ due to chemical reactions or heat sources and sinks. Since the chemical source term $\dot{\omega}_{\alpha}$ is highly non-linear, it is accounted for through a joint sub-grid $s g s$ in conjunction with the Eulerian stochastic field method [7]. The source terms of mass, momentum and relevant scalar, i.e. $\overline{\dot{S}}_{\text {mass }}$, $\overline{\dot{S}}_{\text {mom, } i}$ and $\overline{\dot{S}}_{\alpha}$, are obtained from the volume-averaged contribution of every particle within a specific control volume (see e.g. [8] for more detail).

\subsection{Modelling of Liquid Sprays}

Following the work of Bini [9, 10], a stochastic Markov model is used to account for the effect of the $s g s$ velocity fluctuations on the $p$-th particle over a time $d t$. The evolution of the particle velocity $\boldsymbol{u}_{p}$ is determined by:

$$
d \boldsymbol{u}_{p}=\frac{\overline{\boldsymbol{u}}-\boldsymbol{u}_{p}}{\tau_{p}} d t+\left(C_{o} \frac{k_{s g s}}{\tau_{t}}\right)^{1 / 2} d \boldsymbol{W}_{t}+\left(1-\frac{\bar{\rho}_{g}}{\rho_{l}}\right) \boldsymbol{g} d t
$$

where $\boldsymbol{u}_{p}$ is the particle velocity, $\overline{\boldsymbol{u}}$ and $\bar{\rho}_{g}$ are the filtered gas velocity and density interpolated at the particle location, $\boldsymbol{g}$ is the gravitational acceleration and $\tau_{p}=\frac{\rho_{l}}{\rho_{g}} \frac{4 D_{p}}{3 C_{D}\left|\overline{\boldsymbol{U}}_{s}\right|}$ is the particle response time. The subscripts, $l$ and $g$, describe the liquid and gas phase respectively, $D_{p}$ is the particle diameter and $\left|\overline{\boldsymbol{U}}_{s}\right|$ is the slip velocity. The drag coefficient $C_{D}$ is determined from the drag law of Yuen and Chen [11]. The stochastic contribution includes a model constant $C_{o}$, which is unity in the current work, and the sgs kinetic energy of the gas 
phase $k_{s g s}$ which takes into account the effects of the sgs fluctuations on the particle dispersion through the Wiener process $d \boldsymbol{W}_{t}$. The rate of interaction between particles and turbulence dynamics in the gas phase is represented by the sub-grid time scale as follows [10]:

$$
\tau_{t}=\frac{\tau_{p}^{1.6}}{\left(\Delta / k_{s g s}^{1 / 2}\right)^{0.6}}
$$

The Abramzon-Sirignano model [12] which takes into account the effects of Stefan flow on heat and mass transfer is adopted so that the change of droplet temperature and mass over a time $d t$ may be expressed as:

$$
\begin{gathered}
d T_{p}=-\frac{\dot{m}_{p}}{m_{p} B_{T}^{\prime}}\left(\frac{C_{p, g}}{C_{p, l}}\right)\left(\bar{T}_{g}-T_{p}\right) d t+\left(\frac{h_{f g}}{C_{p, l}}\right) \frac{\dot{m}_{p}}{m_{p}} d t \\
d m_{p}=-\frac{m_{p}}{3 S c_{g} \tau_{p}}\left(S h^{d t}+S h^{s g s}\right) \ln \left(1+B_{M}\right) d t
\end{gathered}
$$

where $T_{p}$ and $\bar{T}_{g}$ are the particle temperature and the filtered gas temperature interpolated at the particle location, $C_{p, l}$ and $C_{p, g}$ are the liquid and gas phase specific heat capacities, $h_{f g}$ is the latent heat of vaporisation and $B_{M}$ and $B_{T}^{\prime}$ are the Spalding mass and heat transfer numbers respectively. The method of Clift et al. [13] is adopted here to calculate the Nusselt and Sherwood numbers, $N u$ and $S h$. The Sherwood number is decomposed into two parts, the resolved contribution $S h^{d t}$ and the unresolved part $S h^{s g s}$ in order to describe the effects of unresolved $s g s$ fluctuations on the temporal evolution of droplet mass. The stochastic contribution to Eq. (7) is determined by:

$$
S h^{s g s} d t=C_{v} S c_{g}^{1 / 3}\left(\rho_{g} \frac{k_{s g s}^{1 / 2} D_{p}}{\mu_{g}}\right)^{1 / 2}\left|d \boldsymbol{W}_{t}\right|^{1 / 2} \tau_{p}^{3 / 4}
$$

where $d \boldsymbol{W}_{t}$ is the increment of the Wiener process and $C_{v}$ is a model constant assumed unity [14].

\subsection{Numerical Investigation}

BOFFIN-LES [15] is an in-house block-structured, parallel, incompressible, variable density code. The gas phase reaction of methanol and air is represented by a reduced reaction mechanism involving 14 reaction steps and 18 chemical species [16] and gas phase sgs turbulence-chemistry interactions are described by the stochastic fields/sgs-pdf equation method. Eight stochastic fields are used.

The computational domain consists of the burner housing and the combustion chamber which extends
33D and 16D in the axial and radial directions respectively with a total number of about 1 million cells and 92 blocks. There are 36 computational cells covering the burner exit. A finer grid resolution with the smallest cell size of $0.17 \mathrm{~mm}$ is applied in the region close to the burner exit where the rapid mixing of air and fuel vapour occurs. The grid size then expands both axially and radially with a maximum expansion ratio of 1.1 and the size of the largest cells becomes $5.2 \mathrm{~mm}$ close to the exit boundaries. The inlet velocity through 24 primary air supply holes is set to $8.12 \mathrm{~m} / \mathrm{s}$ while a small fraction of air with its velocity of $0.42 \mathrm{~m} / \mathrm{s}$ is diverted around the atomizer tip matching the total mass flow rate of air provided in the experimental work. The initial gas phase temperature through all holes and across the entire domain is estimated to be $293 \mathrm{~K}$. Non-reflecting outflow boundary conditions are applied at the exit plane of the computational domain and free slip conditions are used along the sides of the combustion chamber. The injected particle velocity and temperature are $0.325 \mathrm{~m} / \mathrm{s}$ and 293 $\mathrm{K}$ respectively. The flame is ignited using 4 sparks as an enthalpy source to the gas phase within the burner housing.

To approximate the $p d f$ of drop diameter, the following procedure was adopted. Technical data sheets provided by Sono-Tek Corporation [17] show that the typical pdf of drop size for water follows a log-normal distribution with the number median drop size of $32 \mu \mathrm{m}$ and the standard deviation of 0.6. The correlation [18] is used to reproduce the likely pdf of methanol drops. The number median diameter for methanol is calculated to be $23.45 \mu \mathrm{m}$ and the standard deviation of water is retained. In order to approximate the spatial distribution of drops across the injection plane, 5000 equally distributed injection points are prescribed. At each computational time step, droplets are injected randomly at the prescribed points satisfying the overall mass flow rate of liquid.

\section{Results and Discussion}

The simulation was conducted with a time step of 1.0 $\mu \mathrm{s}$. A flow-through time corresponds to $0.0213 \mathrm{~s}$ knowing that the bulk velocity and the length of the geometry under consideration are $20 \mathrm{~m} / \mathrm{s}$ and $0.426 \mathrm{~m}$ respectively. 90,000 time steps (0.09 s) corresponding to 4.2 flow-through times were initially run in order to remove disturbances induced by the initial conditions. All the statistical results presented here were collected over another 80,000 time steps, i.e. 3.8 flow-through times. 


\subsection{General Features and Droplet Behaviour}

Figure 1 presents a contour plot of the instantaneous temperature field with a typical snapshot of individual droplets in order to provide an overall impression of the predicted spray flame structure. The flame attachment to the atomizer tip can be observed and it extends through the contraction section. At the burner exit, a dense column of evaporating droplets is surrounded by a thick envelope flame corresponding to external group combustion. In the upper part of the flame structure, the flame expands slowly in the radial direction and eventually transforms into a turbulent brush flame.

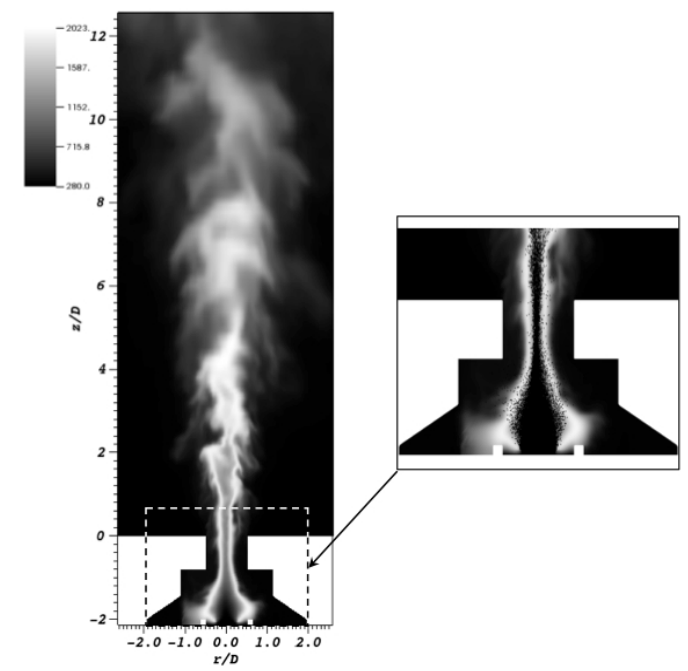

Figure 1: A contour plot of the instantaneous temperature field with droplet motions as well as a close look of the flame structure in the burner housing

The spray properties such as the droplet number density, $D_{10}$ and the $p d f$ of drop size are discussed first as the present configuration is very sensitive to droplet concentrations. Figure 2 presents a comparison of the simulated droplet number density and $D_{10}$ against measurements just above the burner exit $(\mathrm{z} / \mathrm{D}=0.1)$. The computed number density exhibits under-predictions but falls within the range of experimental errors which are as large as $30 \%$ [19] while there is an excellent agreement for the average droplet size.

Figure 3 shows a comparison between the simulated and measured $p d f$ of drop size at $\mathrm{z} / \mathrm{D}=0.1$ and $\mathrm{z} / \mathrm{D}=$ 3.5 along the centreline of the flame. The computed $p d f$ of particle size at $\mathrm{z} / \mathrm{D}=0.1$ shows a minor shift towards the smaller size but is very similar to that specified at the injector. This suggests that the particles travelling within the burner housing may well experience saturation at their surface; the evaporation rate of droplets thus decreases to zero. The simulated droplet size $p d f$ is in
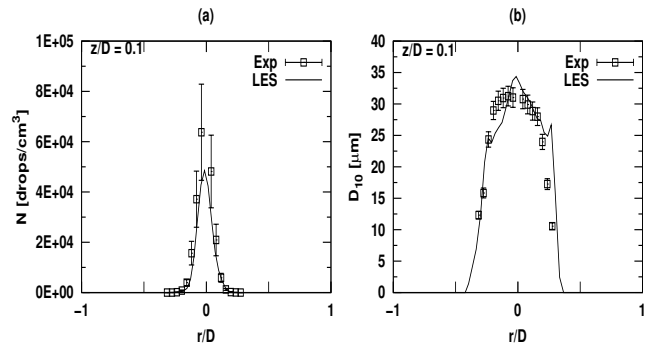

Figure 2: Radial profiles of (a) droplet number density and (b) arithmetic mean diameter at $\mathrm{z} / \mathrm{D}=0.1$

general satisfactory.

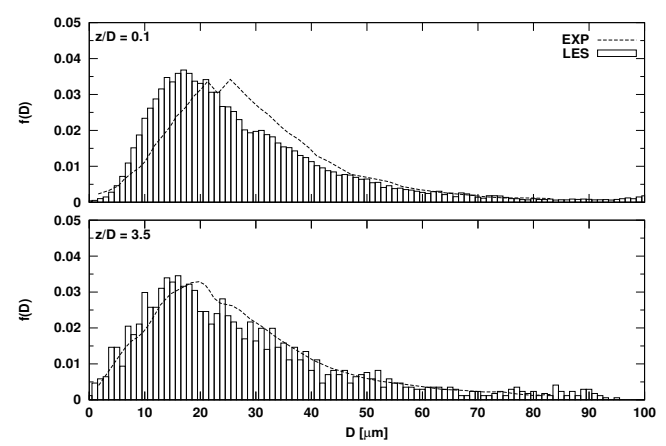

Figure 3: The $p d f$ of droplet size along the centreline of the flame

\subsection{Gas Phase Velocities}

The measured gas velocity statistics are represented by the smallest drops below $8 \mu \mathrm{m}$ while the simulated gas velocities together with those computed by considering all droplets smaller than $12 \mu \mathrm{m}$ are presented. The increase in the size cut-off used as gas tracers is necessary from the numerical point of view to enlarge the sampled population. This increase is justifiable because differences in the average velocities using either $8 \mu \mathrm{m}$ or $12 \mu \mathrm{m}$ are found to be minimal.

Radial profiles of the mean axial velocities are presented in Fig. 4. The overall features such as a distinct depression along the centreline of the flame due to the heavy loading of particles and decelerations of the gas in the axial direction as a result of the jet spreading are well captured from LES simulations. At $\mathrm{z} / \mathrm{D}=0.1$, the predicted droplet axial velocities along the centreline of the flame agree well with experimental data while differences tend to increase away from the centreline. This error is likely to be a result of low droplet concentrations leading to a small number of statistical samples at a given time of the simulation. Nevertheless, it is questionable whether the velocity statistics of small drops can actually be ascribed to those of the gas in the region where the velocity field fluctuates considerably. In the 
upper part of the flame, the magnitude of the simulated droplet velocities is close to the computed gas velocities as well as the measured velocities obtained using gas tracers since particles have enough time to adjust to slow decelerations of the gas. On the other hand, the computed gas velocities show a considerable difference compared with the measured axial velocity away from the centreline in the lower part of the flame.
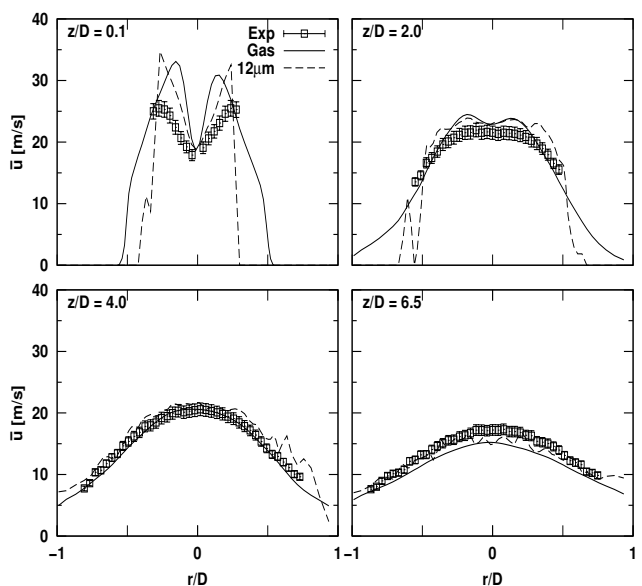

Figure 4: Radial profiles of the mean axial velocities

Figure 5 shows the measured and simulated radial velocities. The computed radial velocities of the smallest drops are again in excellent agreement with those measured experimentally, in contrast the predicted gas phase velocity shows a very different distribution. The numerical work of [5] also reveals that the smallest particles are not suitable to represent the radial velocity components of the gas. The use of small droplets as gas tracers is often justified by Stokes number considerations. The Stokes number is defined as the ratio of the characteristic time of a droplet and a characteristic flow time, i.e. $\mathrm{St}=\frac{\tau_{d}}{\tau_{g}}=\frac{\rho_{l} D_{p}^{2}}{18 \mu_{g}} \frac{1}{\tau_{g}}$. In order for particles to closely follow the gas, the particle response time should be much smaller than the smallest time scale of the flow, typically $\mathrm{St} \ll 1.0$ [20]. In the experiment, the largest Stokes number for an $8 \mu \mathrm{m}$ droplet is estimated to be close to unity [4]. Seeding particles with diameters between 1 and $12 \mu \mathrm{m}$ were injected and tracked to study the variation of Stokes number. The Stokes number of $1 \mu \mathrm{m}$ particles had a maximum of 0.01 near the burner exit whilst that of $12 \mu \mathrm{m}$ particles was between 3 and 4 at the same location. Beyond the burner exit, the Stokes numbers of all the tracked particles tend to decrease with axial distance falling to values well below 1.0. Therefore, the smallest particles are more capable of following the gas flow along the upper part of the flame.

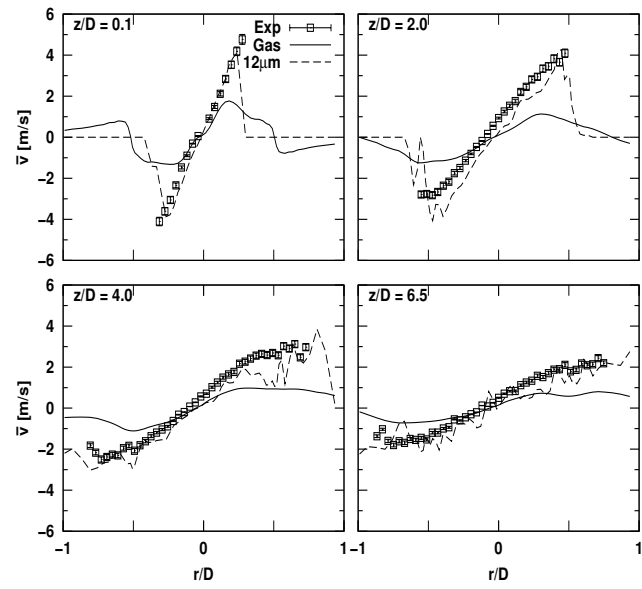

Figure 5: Radial profiles of the mean radial velocities

\subsection{Gas Phase Temperature}

Figure 6 presents a comparison between radial profiles of the simulated and experimental average gas temperature. The peak gas temperature of around $1650 \mathrm{~K}$ is obtained both from LES and experiment. However, there is a large discrepancy between the numerical results and the experimental data. The radial profiles of the simulated gas temperature $(\mathrm{z} / \mathrm{D} \leq 2.0)$ show a depression with values of around $1300 \mathrm{~K}$ along the centreline of the flame while all the measured profiles are bell-shaped with a maximum value of $1650 \mathrm{~K}$ at the centreline. The depression along the centreline has also been reproduced in the simulation of [5]. In the upper part of the flame, the gas temperature profiles are overestimated at all axial locations. Furthermore, the mean temperature from the LES tends to decrease towards the shear layer of the flame and reach the atmospheric temperature. This decreasing trend is not observed experimentally because the technique used therein cannot measure gas temperatures with accuracy below about $600 \mathrm{~K}$ [19].

It is possible that the discrepancy between the results obtained from LES simulations and experiment may be attributable to experimental uncertainty. The Raman thermometry used measures the gas temperature through an averaging technique conditioned on the absence of drops. To make the effects of this conditional average on accuracy of the temperature measurement visible, contour plots of the instantaneous temperature field together with droplet motion close to the burner exit and further downstream are shown in Fig. 7. In the vicinity of the burner exit, the flame is located away from the dense column of drops and the conditional averaging technique will reject most of the laser pulses that are interfered by the presence of drops. The loca- 

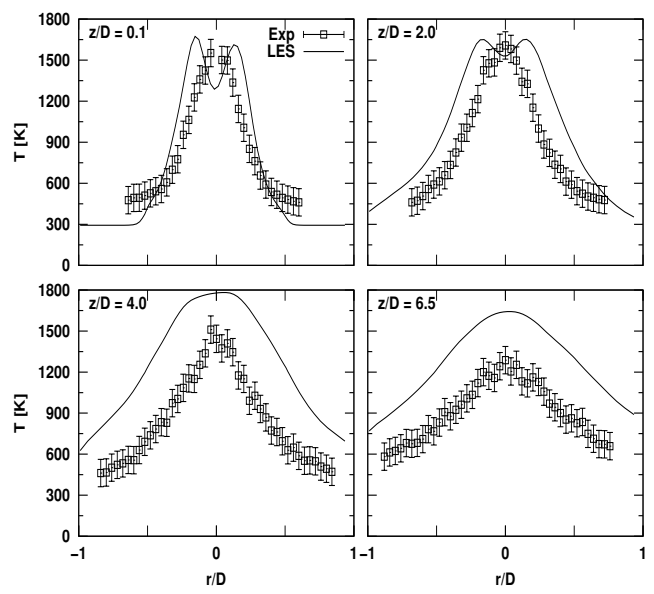

Figure 6: Radial profiles of the mean gas temperature

tions of the heavy loading of particles are found to oscillate considerably in the radial direction and the Raman technique captures a moment when the maximum temperature is situated along the centreline of the combustor. Further downstream, the flame opens up and develops into the turbulent brush flame with separate burning regions. In contrast to the behaviour of particles near the burner exit, they are carried by the large corrugated flame structures. This means that the conditional averaging will lower the gas temperature in the upper part of the flame.

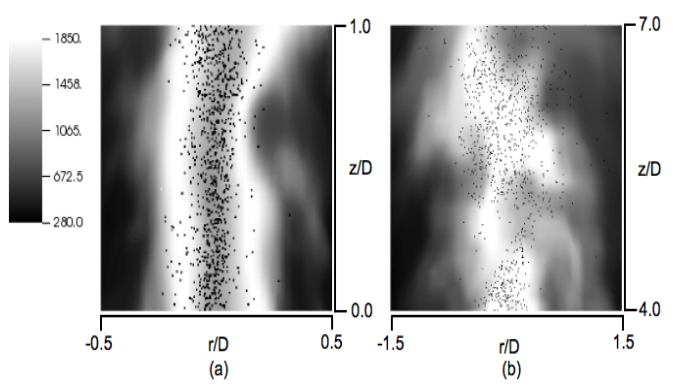

Figure 7: Contour plots of the instantaneous temperature field with the corresponding droplet field (a) in the vicinity of the burner exit and (b) in the upper part of the flame

The conditional gas temperature technique used in the experiment has been mimicked by placing 21 probe volumes with a diameter of $600 \mu \mathrm{m}$ at $\mathrm{z} / \mathrm{D}=0.1$. No computational probes were located in the upper part of the flame because of the significant reduction in the droplet number density in this region. Figure 8 represents the simulated $p d f$ of unconditional and conditional gas temperatures at the centreline of the flame. The $p d f$ of unconditional temperature shows its maximum at around $1250 \mathrm{~K}$ while the conditional measure- ment results in a shift of its maximum $p d f$ towards the higher temperature. The mean unconditional and conditional temperatures are found to be about $1300 \mathrm{~K}$ and $1450 \mathrm{~K}$ respectively at the centreline of the flame.

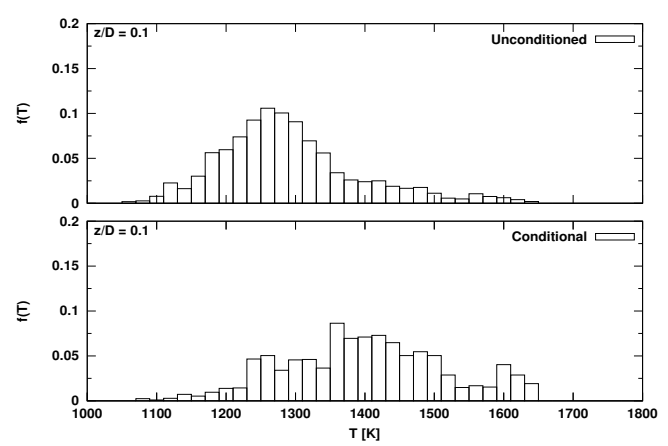

Figure 8: The $p d f$ of unconditional and conditional gas temperature at the centreline of the flame

\subsection{Liquid Phase Velocity Statistics}

Figure 9 shows radial profiles of the average axial velocity for all droplet sizes at several measurement points. In general, the spray axial velocities from LES simulations agree well with those obtained from measurements at all axial positions, indicating that the gas phase velocity fields are well reproduced in the present work. A dip along the centreline of the flame at $\mathrm{z} / \mathrm{D}=$ 2.0 is still observed because the rate of spreading of the jet may be under-estimated.

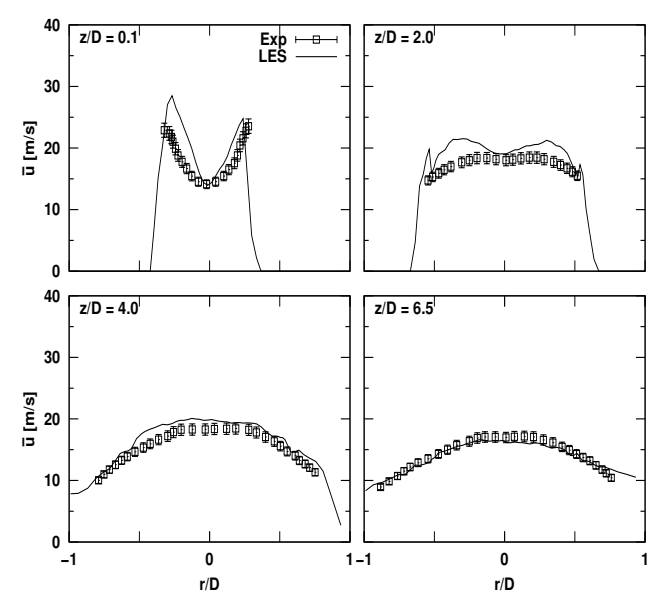

Figure 9: Radial profiles of the mean axial velocities for all droplet sizes

The droplet size and velocity correlations along the centreline of the flame are presented in Fig. 10 to verify the particle dispersion model used in the current work for different drop sizes. At the burner exit, the axial velocity of the smallest particles $(<12 \mu \mathrm{m})$ is close to 
that of the gas phase but shows that larger particles in the size width are less responsive to gas fluctuations. With increasing the axial distance, the difference in the velocity is minimal regardless of the droplet diameter. The computed droplet size-velocity correlation is found to be very close to measurements [19].

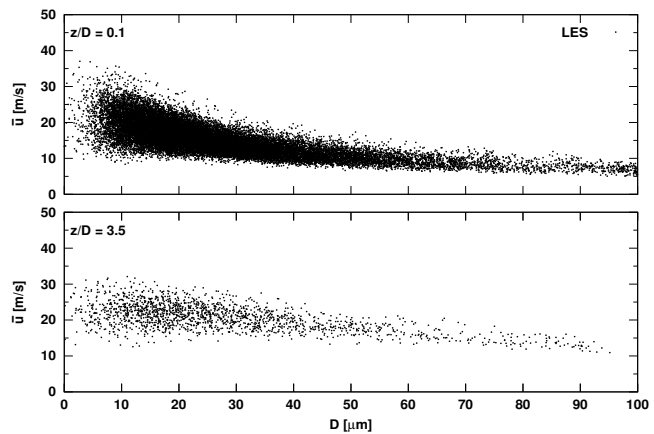

Figure 10: Droplet size-velocity correlations along the centreline of the flame

\section{Conclusions}

In the present investigation, a turbulent methanol spray flame is studied with the aid of LES. The Eulerian stochastic field method coupled with a reduced reaction mechanism involving 14 reaction steps and 18 reactive species are adopted to describe the turbulent combusting gas phase and particle dispersion and evaporation are described in a fully coupled Lagrangian formulation. A reasonably accurate way of estimating the $p d f$ of drop size produced by the particular atomizer is proposed in spite of a lack of experimental information. An analysis of the Stokes number of the small particles suggests that 8 to $12 \mu \mathrm{m}$ particles do not adequately 'follow' the gas flow, especially in the region close to the burner exit. The agreement in the maximum gas temperature at the burner exit is a good indication over the suitability of the implemented evaporation model liberating enough amount of the fuel vapour within the burner housing. The reproduction of gas temperature measurements conditioned on the absence of droplets confirms the presence of a depression in the temperature field along the centreline of the flame. However, it is still uncertain if the large discrepancies further downstream are due to experimental uncertainties or necessitate a further improvement in the evaporation model. The current droplet dispersion model reproduces the spray velocities very accurately.

\section{Acknowledgements}

The work is supported by the 7th Framework Programme of the European Union (FP7/2007-2013) under the Grant Agreement ACP0-GA-2011-265586.

\section{References}

[1] W. P. Jones, S. Lyra, S. Navarro-Martinez, Combust. Flame 159 (2012) 1539-1561.

[2] W. P. Jones, A. J. Marquis, K. Vogiatzaki, Combust. Flame 161 (2014) 222-239.

[3] V. N. Prasad, A. R. Masri, S. Navarro-Martinez, K. H. Luo, Combust. Flame 160 (2013) 2941-2954.

[4] A. N. Karpetis, A. Gomez, Combust. Flame 121 (2000) 1-23.

[5] N. A. Beishuizen, D. Roekaerts, Experiments and Numerical Simulations of Diluted Spray Turbulent Combustion, Springer, 2011, Ch. 5, pp. 133-165.

[6] U. Piomelli, Prog. Aero. Sci 35 (1999) 335-362.

[7] L. Valino, Flow. Turbul. Combust 60 (1998) 157-172.

[8] W. P. Jones, S. Lyra, A. J. Marquis, Int. J. Heat Fluid Flow 53 (2010) 2491-2505.

[9] M. Bini, W. P. Jones, Phys. Fluids 19 (3) (2007) 035104

[10] M. Bini, W. P. Jones, J. Fluid Mech 614 (2008) 207.

[11] M. C. Yuen, L. W. Chen, Combust. Sci. and Tech. 14 (4-6) (1976) 147-154.

[12] B. Abramzon, W. A. Sirignano, Int. J. Heat Mass Transfer 32 (9) (1989) 1605-1618.

[13] R. Clift, J. R. Grace, M. E. Weber, Bubbles, Drops and Particles, Academic Press, New York, 1978.

[14] M. Bini, W. P. Jones, Int. J. Heat Fluid Flow 30 (2009) 471-480.

[15] W. P. Jones, F. di Mare, A. J. Marquis, LES-BOFFIN: User's Guide (2002).

[16] R. P. Lindstedt, M. P. Meyer, Proc. Combust. Inst 29 (2002) 1395-1402.

[17] R. Engle, International Coating Science and Technology Symposium, 2010.

[18] R. J. Lang, J. Acoust. Soc. Am 34 (1962) 6.

[19] A. N. Karpetis, A. Gomez, J. Fluid Mech 397 (1999) 231-258.

[20] D. E. Rosner, Transport Processes in Chemically Reacting Flow Systems, Dover Publications, New York, 2000, p. 314. 